\title{
Prediction of thermomechanical behavior of acrylonitrile butadiene styrene using a newly developed nonlinear damage-reliability model
}

\author{
A. En-naji \\ Laboratory of Control and Mechanical Characterization of Materials and Structures, National Higher School of Electricity and \\ Mechanics, BP 8118 Oasis, Hassan II University, Casablanca, Morocco \\ abdenaji14@gmail.com,arrahma78@yahoo.fr
}

\section{N. Mouhib}

Laboratory of Control and Mechanical Characterization of Materials and Structures, National Higher School of Electricity and Mechanics, BP 8118 Oasis, Hassan II University, Casablanca, Morocco

Higher Institute of Maritime Studies ISEM, Casablanca, Morocco

\section{H. Farid}

Laboratoire de Nanotechnologie et Bioplasturgie, Université du Québec en AbitibiTémiscamingue UQAT, boulevard de l'Université, Rouyn-Noranda, Québec J9X 5E4, Canada

\section{El Ghorba}

Laboratory of Control and Mechanical Characterization of Materials and Structures, National Higher School of Electricity and Mechanics, BP 8118 Oasis, Hassan II University, Casablanca, Morocco

\footnotetext{
ABSTRACT.The aim of this work was to evaluate the influence of temperature on the mechanical behavior of an amorphous polymer, namely acrylonitrile butadiene styrene (ABS), based on a series of uniaxial tensile tests on smooth specimens at different temperatures.

The results demonstrate that the behavior of the polymers is strongly dependent on the temperature. Its influence on the physical characteristics during the study of polymer behaviors cannot be denied, particularly when the processes of shaping are investigated, which require significant contributions of heat and mechanical effort. For this reason, this study consists of predicting the evolution of ABS damage in two main zones.

The first is the industrial zone, in which the configuration of macromolecular chains is largely immobile, and the temperature is below the glass temperature $\left(\operatorname{Tg}=110^{\circ} \mathrm{C}\right)$. In this zone, a damage model based on the obtained experimental results allowed us to determine three stages of damage evolution, and then to specify the critical fraction of life, at which the material becomes unstable and defective, for the purpose of predictive maintenance.

The second zone is that of thermoforming, in which the temperature is above the glass temperature, Tg. In this zone, the macromolecular chains tend to
}

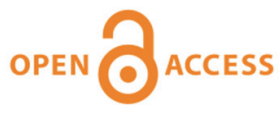

Citation: En-naji, A., Mouhib, N., Farid, H., ElGhorba, M., Prediction of thermomechanical behavior of acrylonitrile butadiene styrene using a newly developed nonlinear damage-reliability model, 49 (2019) 748-762.

Received: 08.05.2019

Accepted: 17.05 .2019

Published: 01.07.2019

Copyright: (C) 2019 This is an open access article under the terms of the CC-BY 4.0, which permits unrestricted use, distribution, and reproduction in any medium, provided the original author and source are credited. 
move more freely as the temperature increases. The same damage model was adopted to follow the flow process according to the fraction of life that represents the critical material parameter.

This study also includes a comparison between the static (experimental) damage models and unified theory (theoretical) damage models.

KEYWORDS. ABS; Damage; Flow; Stress; Reliability; Temperature; Thermomechanical behavior; Tensile testing.

\section{INTRODUCTION}

he prediction and evaluation of material thermomechanical behavior have long been serious concerns. Thus, numerous models have been developed in to quantify the damage caused by steel materials, with reference to either linear or nonlinear models. Miner (1944) was the first author to formulate a fatigue damage law mathematically, and its linear formulation of the damage as a function of the fraction of the total work absorbed by the material made it a simple and obvious law to use. Other authors, including Quoc et al. (1971), proposed modeling of the damage depending on the loading conditions, stress level, and material characteristics. The models of Henry (1955), Lemaitre and Chaboche (1979), and Gatt (1961) can also be cited as representative damage models.

Zgoul and Habali (2008) applied these different theories to steel materials and their alloys. To adapt these theories to polymer materials, we applied these to anacrylonitrile butadiene styrene (ABS) thermoplastic polymer. A substantial amount of research has been conducted on this material to quantify its mechanical behavior. Gonzalo et al (2015) evaluated the effect of the ultrasonic fatigue endurance of polymeric material ABS, and the general mechanisms of crack initiation and propagation, when specimens were tested by being immersed in water and oil, to limit the temperature increase induced by the high mechanical vibration of ultrasonic fatigue testing. Joseph et al. studied the effects of the toxicity of ABS combustion products. Bohatka et al (1995) evaluated the damage formation differences during fatigue crack propagation in ABS polymer, using tests both fulfilling and not fulfilling the linear elastic fracture mechanics requirements, which related to differences in the crack propagation behavior through the crack layer. Boldizar et al. (2003) studied the degradation of ABS during repeated treatments and accelerated aging, whereby the ABS material was subjected to a series of six combined cycles of extrusion and air aging as well as high temperatures, and observed changes in the tensile and flow properties. From the second to the sixth cycle, the elongation at break decreased considerably owing to aging, while it increased as a result of the extrusion step. Shen et al. (1966) and Merah et al. (2003) studied the temperature and weld-line effects on the mechanical properties of chlorinated PVC(CPVC). They investigated the effects of temperatures ranging from -10 to $70^{\circ} \mathrm{C}$ and injectionmolded weld lines on the mechanical properties of CPVC used in pipe fittings. In their studies, they demonstrated that brittle fracture occurred at temperatures below room temperature, while ductile failure occurred at temperatures above $23^{\circ} \mathrm{C}$. At temperatures below $50^{\circ} \mathrm{C}$, the fracture stress increased in small amounts. A significant increase in the fracture deformity occurred between 50 and $70^{\circ} \mathrm{C}$. Plastic deformation at these high temperatures was accompanied by considerable shrinkage.

Our work is based on thermomechanical characterization of the material. Several series of tests were carried out in a temperature range of 25 to $170^{\circ} \mathrm{C}$, passing through the glass transition temperature $(\mathrm{Tg})$ close to $110^{\circ} \mathrm{C}$. For each temperature value, the curves relating to the thermomechanical characteristics such as the stress, elastic modulus, and elongation were plotted to identify the measurements in question for analyzing the material thermomechanical behavior. Thereafter, an extensive analysis of the damage was performed using static damage-reliability modeling. The results of comparing the damage of three parameters, namely the ultimate stress, elastic modulus, and elongation, according to the fraction of life, allowed us to predict the thermomechanical behavior of this polymer in the industrial and thermoforming zones.

\section{MATERIAL AND EXPERIMENTAL METHODS}

he material used in this work was ABS, which is an amorphous polymer produced by emulsification or bulk polymerization of acrylonitrile and styrene in the presence of polybutadiene. 
The tensile specimens, illustrated in Fig.1, were prepared according to the ASTM Standard D638-03 [14] method of tests for the tensile properties of plastics.

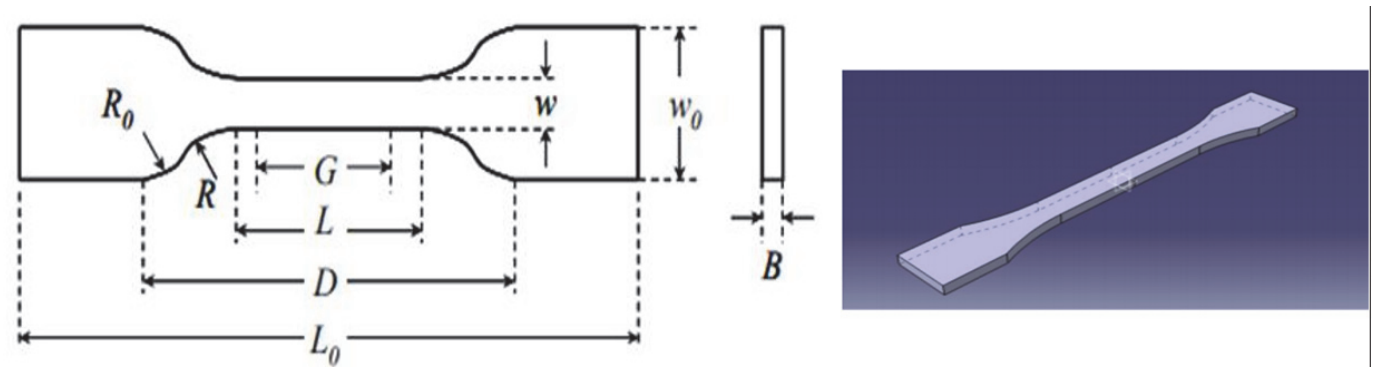

Figure 1: Dimensions of specimens according to ASTM D638-03[14].

\begin{tabular}{ccc}
\hline Symbol & Description & Size (mm) \\
$\mathrm{L}_{0}$ & Total lengh & 75 \\
D & Initial distance between jaws & 42 \\
L & Lengh of the calibrated part & 25 \\
G & Lenth between landmarks & 20 \\
W & With of the calibrated & 4 \\
R & Small radius of curvature & 8 \\
W0 & Widths at the ends & 14 \\
B & thikness & 2 \\
\hline
\end{tabular}

Table 1: The dimensions of dumbbell specimen for tensile test.

Fig.2 illustrates the evolution of the stress applied to the specimens (MPa) as a function of the deformation, $\mathcal{E}(\%)$. The general appearance of this curve exhibits ductile behavior.

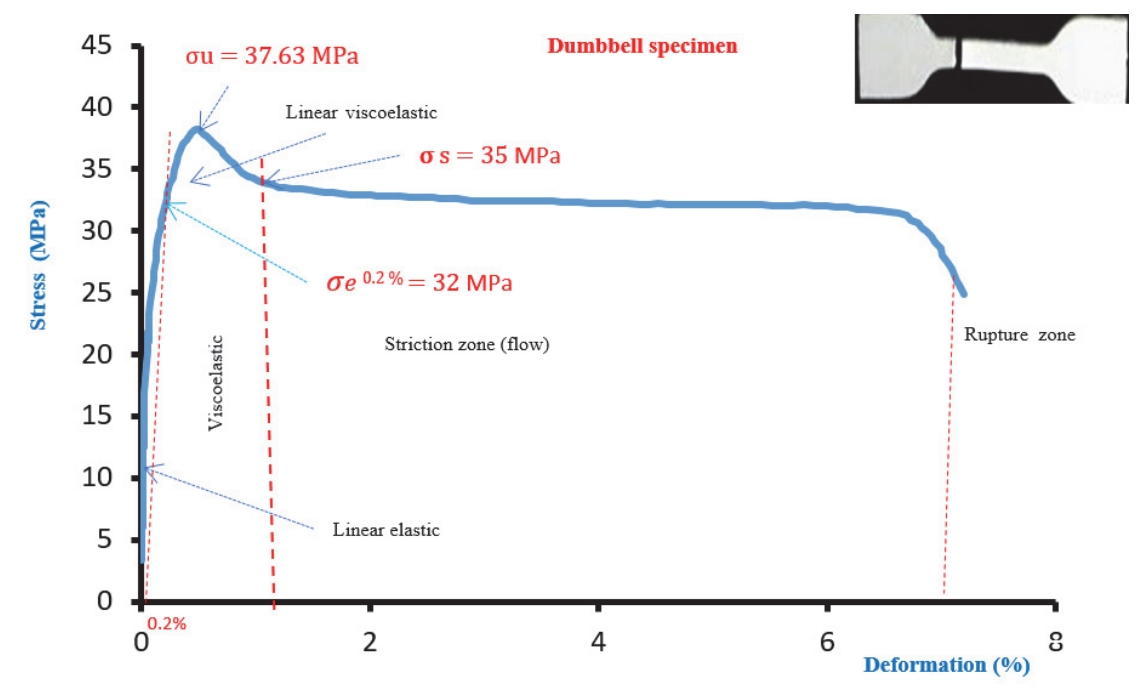

Figure 2: Tensile stress-Deformation curvedumbbell specimen.

It can be observed from Fig. 2 that the curve includes four zones, each of which reveals a particular mechanical behavior of the material (ABS) during the tensile test.

Zone 1 (linear): this is the reversible elastic deformation of the material owing to the amorphous phase. 
Zone 2: the force decreases, indicating the beginning of the constriction, which corresponds to heterogeneous deformation of the material.

Zone 3: the constriction zone increases along the specimen until stabilization.

Zone4: the stretching force increases, and the deformation again becomes homogeneous owing to the structural hardening linked to the orientation of the macromolecular chains in the stretching direction; the material febrile fraction increases until rupture.

The results presented in Fig. 2 enabled us to determine the mechanical properties of the studied material.

These properties include the elastic limit, elasticity modulus, and breaking stress, which are provided in Tab. 2.

\begin{tabular}{cccc}
\hline Young's modulus & Poisson's ratio & Elastic limit & Ultimate stress \\
$\mathrm{E}=2.08 \mathrm{GPa}$ & $v=0.3$ & $\sigma_{e}=32 \mathrm{MPa}$ & $\sigma_{U}=37.63 \mathrm{MPa}$ \\
\hline
\end{tabular}

Table 2: Characteristics of ABS material.

The mechanical properties of polymers are strongly dependent on the temperature. For this reason, the tensile test was carried out under different temperature conditions (elevated temperature tensile testing). The thermal enclosure required for this purpose was controlled by testXpert II. The enclosure had a temperature range of -80 to $+250^{\circ} \mathrm{C}$ (Fig. 3 ).

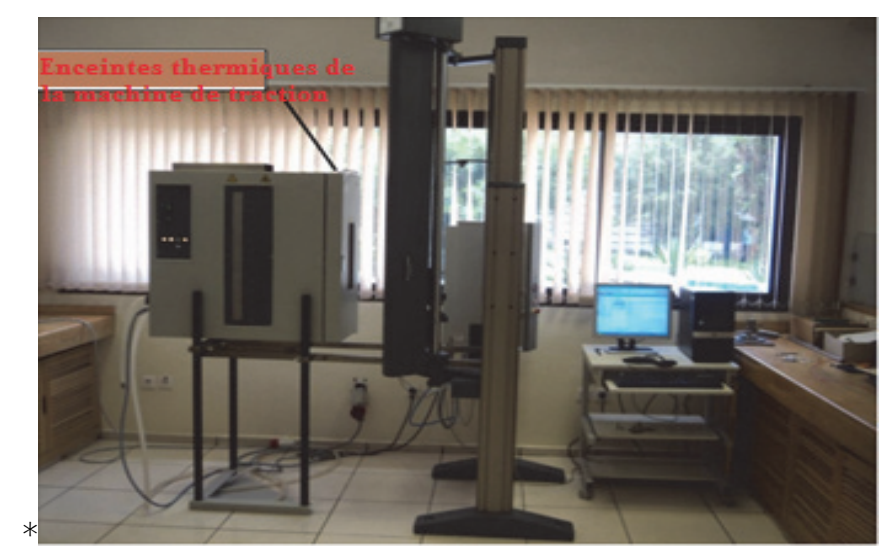

Figure 3: Thermal enclosure assembly with tensile test machine.

Polymers exhibit very precise temperature possibilities for the movements of radicals or pieces of molecular chains; that is, the polymer mechanical behavior will be affected (Fig. 4).

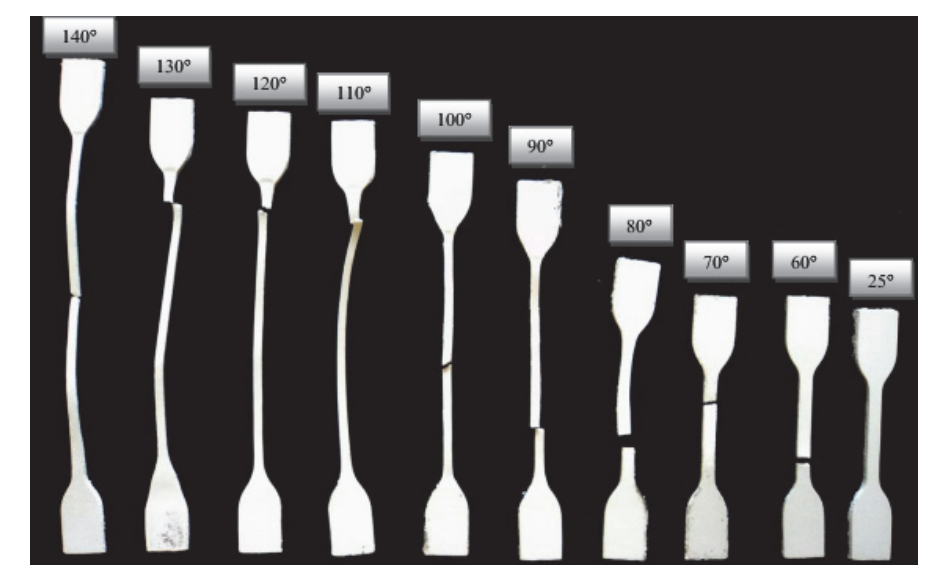

Figure 4: Test specimens subjected to tensile tests as function of temperature. 


\section{RESULTS AND DISCUSSION}

\section{Temperature Effect on mechanical Behavior for ABS}

1 o take into account the influence of the temperature on the ABS mechanical properties, several series of tests were carried out on dumbbell specimens in a temperature range from $25^{\circ} \mathrm{C}$ (laboratory temperature) to $170^{\circ} \mathrm{C}$, through the glass transition temperature $\mathrm{Tg}=110^{\circ} \mathrm{C}$. The variations in the ultimate stress, elastic modulus, and elongation according to the temperature are presented in Figs. 5, 6, and 7, respectively.

Several features of these figures are worth noting: for example, increasing the temperature produces a decrease in the elastic modulus, a reduction in the tensile strength, and an enhancement in the elongation.

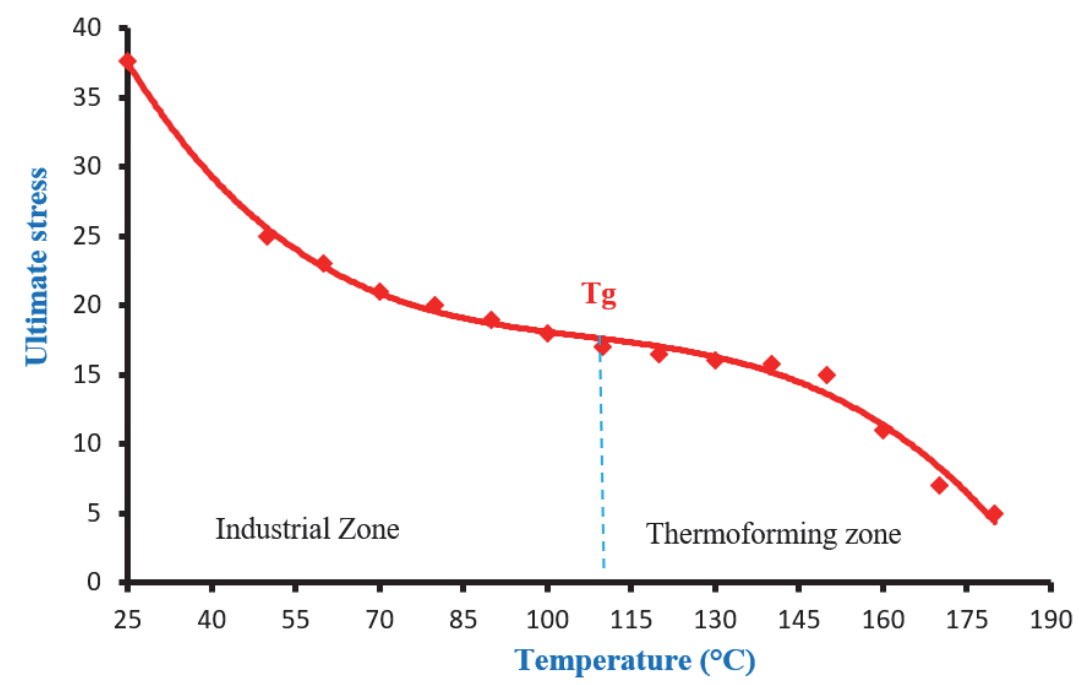

Figure 5: Loss of ultimate stress according to temperature.

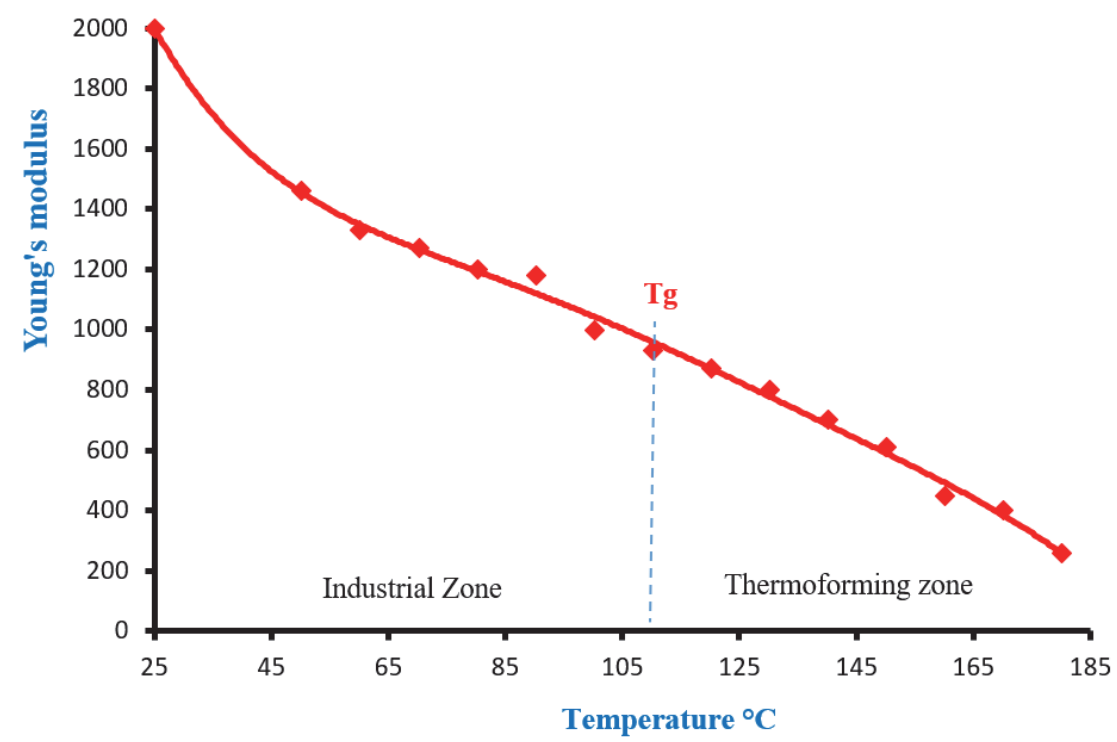

Figure 6: Loss of Young's modulus according to temperature.

We can observe the existence of two zones: zone I corresponds to a temperature that lies between the laboratory temperature $\mathrm{Ta}=25^{\circ} \mathrm{C}$ and glass transition temperature $\mathrm{Tg}=110^{\circ} \mathrm{C}$. Corresponding to a rigid (glass) state, the molecules of the amorphous part are deformed (industrial zone). Zone II is above the glass temperature and corresponds to a transition state in which chain movements become possible, allowing for greater and easier deformations. The mechanical properties 
decrease in this phase; that is, that the mechanical behavior of the polymers is affected. This is a non-industrial zone (which could be exhibited as a thermoforming zone).

Furthermore, Figs. 5 and 6 indicate a sudden decrease in both the ultimate stress and Young's modulus with an increase in temperature, which accelerates once the Tg range is exceeded. However, Fig. 7 illustrates a pseudo-linear variation in the elongation as a function of the temperature, for temperatures below $\mathrm{Tg}$, while the evolution retains a slight slope with an elongation above $10 \%$. When approaching $\mathrm{Tg}$, the elongation values increase more significantly to the melting zone; in this case, we refer to rubbery behavior, which corresponds to a flow owing to the disentanglement of macromolecular chains [15].

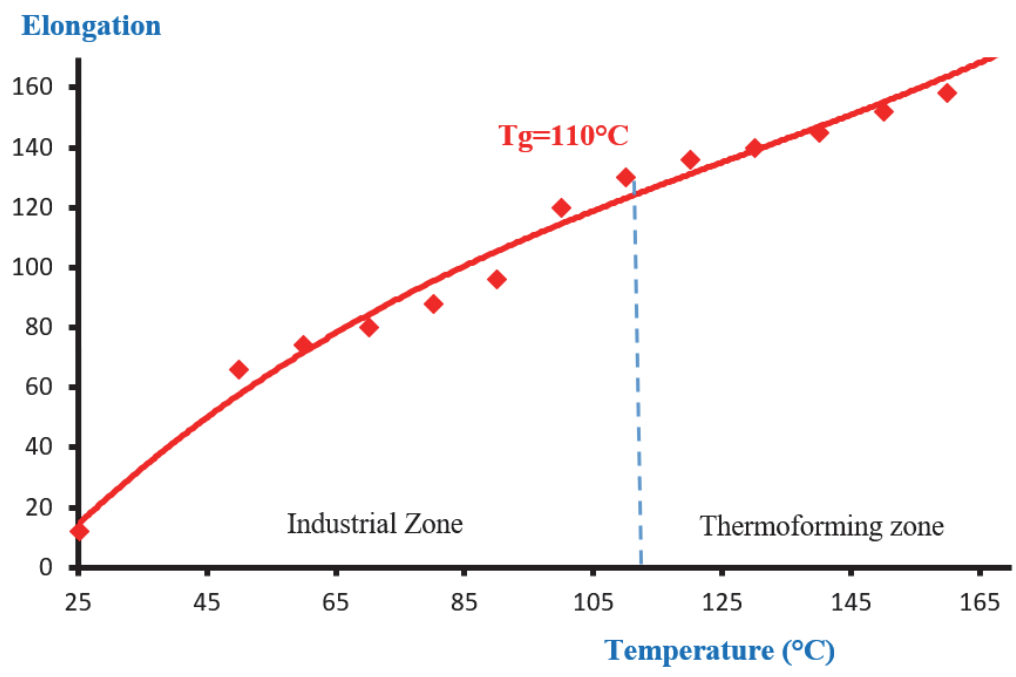

Figure 7: Evolution of the elongation modulus according to the temperature.

\section{Thermomechanical behavior of $A B S$ material in the industrial zone $\left(25^{\circ} \mathrm{C}\right.$ to $\left.110^{\circ} \mathrm{C}\right)$}

\section{Adimensional loss of mechanical properties by fraction of life}

In order to normalize the values of the ultimate stress, Young's modulus, and elongation, the ratio Xur/Xu was examined as a function of the fraction of life [16].

Xur: Either residual ultimate stress(бur), residual ultimate Young's modulus (Eur) or residual ultimate elongation (Lur)

$\mathrm{Xu}$ : Either ultimate stress $(\sigma \mathrm{u})$, ultimate Young's modulus $(\mathrm{Eu})$ or ultimate elongation $(\mathrm{Lu})$

The fraction of life $\beta$ is expressed for the curves of the stress and Young's modulus, as follows[16]:

$$
\beta=\frac{\mathrm{Ti}-\mathrm{Ta}}{\mathrm{Tg}-\mathrm{Ta}}
$$

where:

Ta : ambient temperature ;

Ti : Instantaneous temperature ; and

$\mathrm{Tg}$ : glass temperature

However, we observe that the elongation increases gradually with an increase in the temperature, for which we used the fraction of life[16]

$$
\beta^{\prime}=1-\frac{\mathrm{Ti}-\mathrm{Ta}}{\mathrm{Tg}-\mathrm{Ta}}
$$

The results of the adimensional loss of the three studied mechanical properties Xur/Xu by the fraction of life are presented in Fig.8.

Firstly, we can observe that the curves of the stress and Young's modulus of the ABS decrease as the fraction of life increases: virgin $\mathrm{ABS}$ at room temperature (from $25^{\circ} \mathrm{C}$ ) has an ultimate stress of $32 \mathrm{MPa}$ and a Young's modulus of $2000 \mathrm{MPa}(\sigma u r / \sigma u$ $=$ Eur $/ \mathrm{Eu}=1)$. These adimensional ratios decrease remarkably as a function of the fraction of life $\beta$, until the same value 
of 0.5 is obtained for a fraction of life equal to 1 , which has a critical material temperature, beyond which the material exhibits no significant resistance. The shape of the curves in the interval $\beta=0$ to 0.4 with $\mathrm{T}=44^{\circ} \mathrm{Cappears}$ to indicate that the molecular chains are deformed: at very low relative evolution of the mobility of the molecular chains, the material mechanical behavior resembles that of fragile material. The increase in temperature may particularly increase the mobility and deformation of the molecular chains in the polymer to subsequently generate $\beta>0.4$, a plasticizing phase that is more decisive for the loading amplitude. This general pattern of the deterioration of the polymers under the temperature effect makes it possible to explain the faster failure rate of the test specimens.

However, the shape of the elongation curve in the interval $\beta^{\prime}=0$ to 0.5 indicates that the increase in temperature exhibits an evolutionary effect of the microcracking manifested in the ABS material.

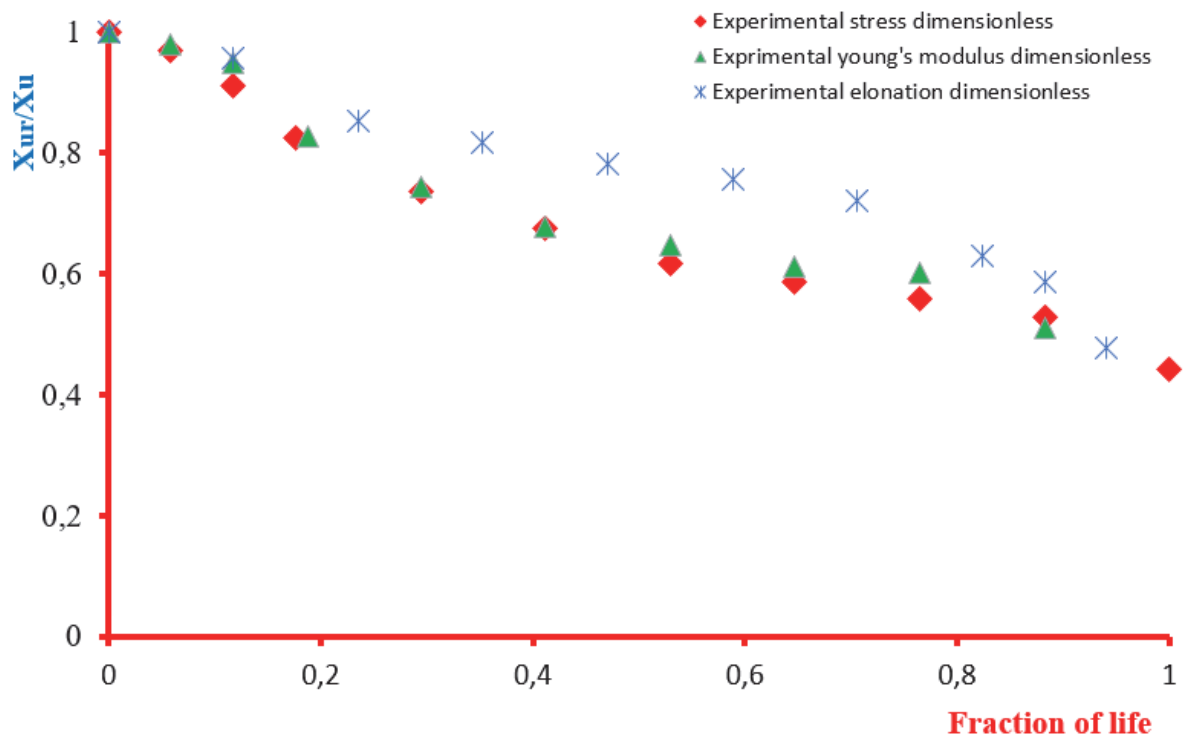

Figure 8: Adimensional loss of ABS mechanical properties.

\section{Comparison between theoretical and experimental adimensional loss of mechanical properties}

By means of analogy with the modified Bui Quoc law (1971)[17] and Bathias (2013), a mathematical expression describing the rate of adimensional loss of the mechanical properties as a function of the fraction of life was exploited to provide a benchmark for comparison between the present theory and experimental results. The loss rate is represented by the following mathematical expression[17]:

$$
\frac{X_{u r}}{X_{u}}=\left[\gamma-\frac{1}{\frac{1-\beta}{\gamma-1}+\frac{\beta}{\gamma-\left(\frac{\gamma}{\gamma_{u}}\right)^{m}}}\right]^{1 / m}
$$

in which

Xur: either residual ultimate stress (бur), residual Young's modulus (Eur) or residual elongation (Lur);

Xu:eitherultimate stress $(\sigma \mathrm{u})$, ultimateYoung'smodulus $(\mathrm{Eu})$ or ultimateelongation $(\mathrm{Lu})$;

$\gamma \mathrm{u}$ : either non-dimensional stress $\left(\frac{\sigma \mathrm{u}}{\sigma \mathrm{a}}\right)$, non-dimensional Young's modulus $\left(\frac{\mathrm{Eu}}{\mathrm{E}_{\mathrm{a}}}\right)$, or non-dimensional elongation $(\mathrm{Lu} / \mathrm{La})$;

$\gamma$ : instantaneous non-dimensional endurance limit : either $\frac{\text { our }}{\sigma a}, \frac{\text { Eur }}{\mathrm{E}_{\mathrm{a}}} \operatorname{or}(\mathrm{Lur} / \mathrm{La})$; 
$\sigma u$, Eu : endurance limit of the virgin material and Lu: Value of the elongation modulus at critical temperature; our, Eur, Lur :residual ultimate stress, Young's modulus, and residual elongation of material at different temperatures; бa, Ea: values of stress and Young's modulus directly before rupture; and $\mathrm{m}$ : a material parameter, with $\mathrm{m}=1$ for amorphous polymers, according to [18].

Graphical representations of the theoretical adimensional loss of the mechanical properties compared to the experimental loss are presented in Fig. 9 below.

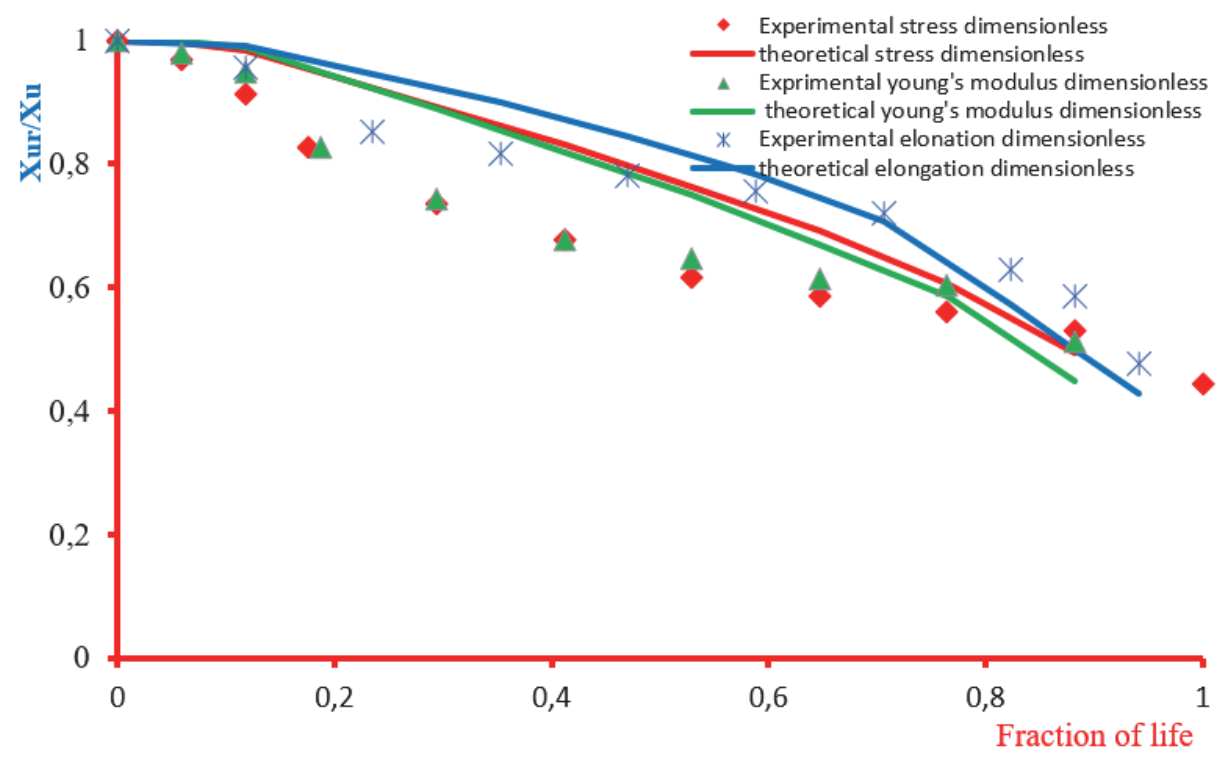

Figure 9: Theoretical and experimental adimensional loss of mechanical properties

Ibe observed from the comparison in Fig. 9 that the increase in temperature induces a greater reduction in both the theoretical and experimental residual stress and elastic modulus, and an evolution of the ABS elongation. Moreover, it is observed that the theoretical curves are situated slightly above the experimental curves. For a low $\beta$ value $(\beta<0.2)$, the theoretical curves agree with the experimental curves. For a mean $\beta(0.2<\beta<0.7)$, the experimental curves tend to overestimate the resistance loss. However, this tendency is reversed for a value close to 1, and the theoretical points are slightly lower than the experimental ones. Consequently, the results demonstrate that the deviation between the theoretical and experimental curves is more or less remarkable at the beginning of the damage, following which the deviation narrows and the curves decrease proportionally in value directly before the break. It can be concluded that the modified Bui Quoc model accurately describes the loss of the adimensional mechanical properties of the ABS material and can be used in the case of static loading coupled with temperature variations.

\section{Quantification of Damage}

Damage is a physical phenomenon [19] that can be determined quantitatively and qualitatively by measuring certain physical properties, particularly mechanical properties such as the yield stress, resistance to rupture, Young's modulus, and elongation modulus.

The static damage model consists of determining the evolution of the thermomechanical characteristics of the ABS material as a function of the fraction of life. The static damage (D), based on variations in the stress, Young's modulus, and elongation, was developed to predict the damage evolution and impact of artificial pre-loading, which is represented by the temperature variation.

During the test, we observed the phenomenon of damage between the virgin state at an ambient temperature until the state of the test piece at a glass temperature of $110^{\circ} \mathrm{C}$, by measuring the $\mathrm{ABS}$ mechanical properties for each temperature variation. The static damage model D is represented in Eqn. (4) [20-23]:

$$
D=\frac{1-\frac{X u r}{X u}}{1-\frac{X a}{X u}}
$$


with:

Xur: either residual ultimate stress ( $\sigma u r)$, residual ultimate Young's modulus (Eur) or residual ultimate elongation (Lur); Xu: either ultimate stress $(\sigma u)$, ultimate Young's modulus $(\mathrm{Eu})$ or ultimate elongation $(\mathrm{Lu})$; and

Xa:either stress $(\sigma a)$, Young's modulus $(\mathrm{Ea})$ or elongation $(\mathrm{La})$ of the material directly before the end of its life.

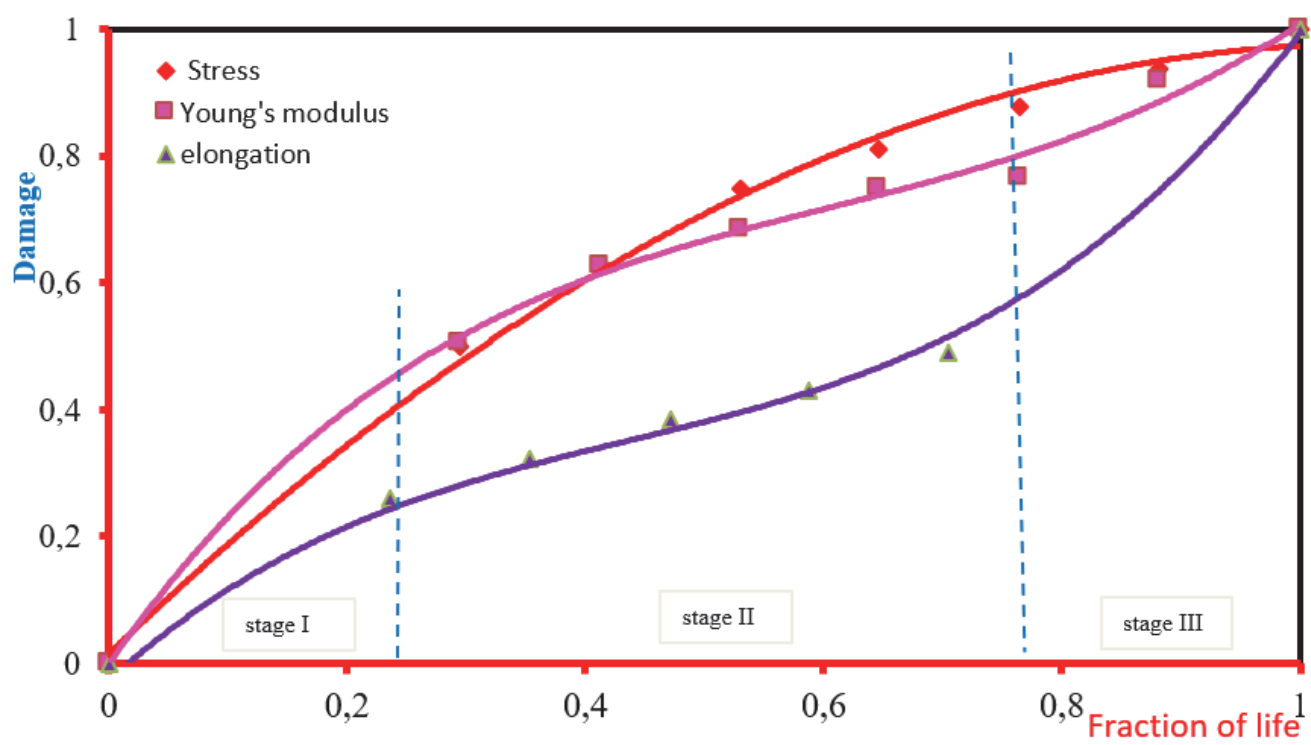

Figure 10: Evolution of the static damage depending on life of fraction

Fig. 10 illustrates the evolution of the normalized experimental damage as a function of the fraction of life $\beta$, using one of the studied mechanical properties each time. The damage process is schematized by a concave curve, which means that the damage accelerates towards the end of the material life at $\mathrm{D}=1$. The increase in damage means an increase in the elongation, and a decrease in the stress and elastic modulus in the static tensile tests of the ABS specimens. This is damage with appreciable irreversible deformations, which reduces the material ultimate strength.

The damage briefly increases from zero (laboratory temperature) to its critical glass temperature. The damage shape and level at the end of the material life $(\mathrm{D}=1, \beta=1)$ provide this experimental damage model with certain credibility, which is consistent with the literature in an equivalent study on metals. Having determined the normalized damage, we have an experimental reference necessary for the validation of theoretical models or other measurement approaches for experimental damage. It is remarkably interesting to be able to correlate the damage process to the three stages of damage. By observing the damage curves represented by the stress and Young's modulus, we can note the following characteristics:

At the initiation of the damage, namely the end of the stage I, in which the fraction of life $\beta=30 \%$, the damage increases in a concave and slow manner.

In the progressive zone, namely stage $\Pi$, which is in the interval of $\beta=[30 \%, 75 \%]$, the damage progressively evolves to 0.8 .

At the moment of sudden propagation (stage $\amalg$ ), in which the fraction of life $\beta>75 \%$ for $\mathrm{D}=0.8$, the damage accelerates considerably.

The purple appearance illustrates the evolution of the static damage of the elongation as a function of the fraction of life $\left(\beta^{\prime}=1-\beta\right)$. Although the damage scenario has been established as the measure of the residual elongation, we also distinguish three stages:

- Stage 1: the damage evolves linearly between 0 and 0.3 for fractions of life $\beta^{\prime}<0.36$.

- Stage 2: the damage increases gradually from 0.3 to 0.5 for fractions of life $0.3<\beta^{\prime}<0.7$, and the temperature effect appears clearly as the temperature increases, while the damage within the material evolves significantly.

- Stage 3: for $\beta^{\prime}>0.7$ the damage accelerates rapidly until rupture.

\section{Relationship betweenStatic Damage and static Reliability}

When a system is in service under static stress, its physical properties undergo progressive degradation. It is often necessary to reduce the probability of sudden failure. Consequently, reliability assessment becomes indispensable in any study of the 
mechanical behavior of the components. In this section, we present a reliability study to reduce the probability of sudden failure.

The reliability $\mathrm{R}$ is a statistical parameter, which follows the evolution of the material deterioration. The relationship between these two parameters can be expressed as follows: $\mathrm{R}(\beta)+\mathrm{D}(\beta)=1$ (5) [21].

Using Eqn. (5) to plot the different reliability curves corresponding to the various parameters studied, the following figures illustrate the variations in the reliability and damage as a function of the fraction of life in the industrial zone. We note that the damage always evolves in the opposite direction to the reliability as the temperature increases.

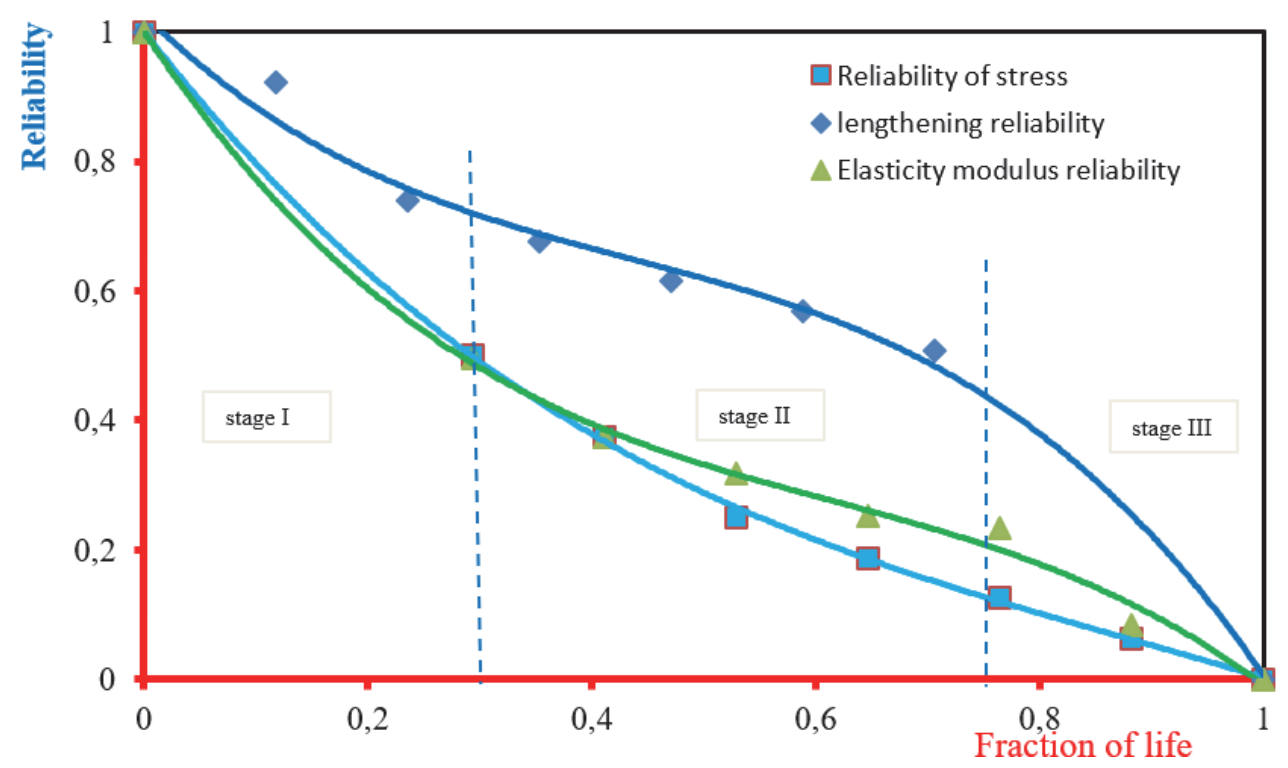

Figure 11: Evolution of damageand reliability as function of fraction of life .

The present work provides the opportunity to discuss the mechanical behavior of a well-known polymer that is extensively used in industrial areas; specifically, in the manufacture of cars. To facilitate quality control without the need for an expensive dynamic test, we conducted a study to compare the mechanical characteristics with the aid of elevated temperature tensile testing. The analysis of the static and thermic tests allowed us to evaluate the negative effects of the increase in temperature on the ABS mechanical behavior more significantly. Indeed, the curves in Fig. 11illustrate that the stress and Young's modulus parameters exhibit comparable damage, regardless of the fraction of life. To overcome the challenges of dynamic tests, we modified the unified theory controlled by elongation, to consider a new parameter relating to the mobility of molecules and the constriction of material. Although the results obtained from the three parameters are in harmony, the use of elongation remains less precise and exhibits a phase shift compared to the other parameters (Young's modulus and stress).

\section{Thermomechanical behavior of ABS material in thermoforming zone (non-industrial zone, $T>T g$ )}

The degradation of the mechanical properties is always remarkable. The elastic stress, ultimate stress, tensile strength, and elasticity decrease more significantly as the temperature increases, and the material begins to deform permanently. The intrinsic softening zone corresponds to the beginning of the nonlinear distortion (this softening of the plastic flow threshold is mainly owing to the change in the material microscopic structure), and this deformation continues with a remarkable increase in temperature. Thereafter, it falls towards the point at which it stabilizes and remains constant with an increasing elongation. The plastic deformation is owing to the material rubbery state, and this deformation is localized on the section of the sample in a region known as the curing zone.

The rubbery state is a result of the amorphous phase, and exists in practically all polymers; it generally begins around the glass transition temperature and is limited by the melting temperature or thermal decomposition temperature.

In the amorphous phase, the molecular organization is constantly changed by displacement of the molecules, owing to thermal activation or the applied stress (constraint). This state change with respect to the vitreous state described above is accompanied by rupturing of certain weak bonds (Van der Waals) between molecules by means of thermal agitation, and a significant increase in the polymer volume. This results in greater ease of movement of the molecules, so that the very low molecular mobility in the vitreous state increases with an increasing temperature. 
As mentioned previously, the rubbery state is owing to the amorphous phase; it is a consequence of the molecular mobility, which allows the chains to be unfolded in the direction of the applied stress, thereby causing deformation. When this constraint is released, it returns to the initial state. A higher cross-linking ratio results in a greater length of the chains between two cross-linking nodes. However, if the nodes of the cross-links are removed, the memory of the initial state disappears, as well as the reversibility of the deformation.

In the following, similar to the damage study in the industrial zone, the flow evolution in the thermoforming zone is reported using the same three parameters. In this zone, we define the fraction of life $\theta$ for the curves of the stress and Young's modulus, as follows [16]:

$$
\theta=\frac{\mathrm{Ti}-\mathrm{Tg}}{\mathrm{Tc}-\mathrm{Tg}}
$$

where:

$\mathrm{Tg}$ :glass temperature;

Tc :critical temperature; and

Ti :instantaneous temperature.

Moreover, for elongation, we use the following expression for the determination of the fraction of life [16]:

$$
\theta^{\prime}=1-\frac{\mathrm{Ti}-\mathrm{Tg}}{\mathrm{Tc}-\mathrm{Tg}}
$$

\section{Adimensional loss of mechanical properties by fraction of life}

The variation in the ABS mechanical properties as a function of the fraction of life is illustrated in Fig.12.

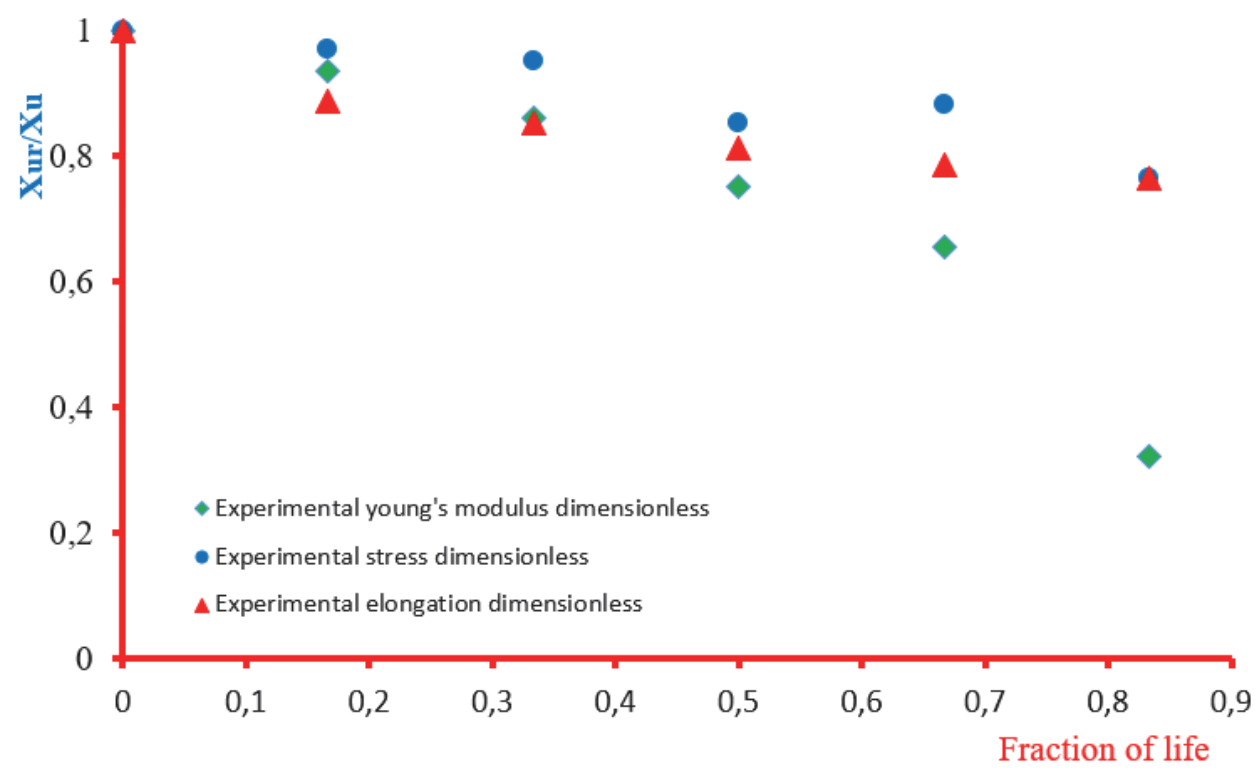

Figure 12: Adimensional loss of ABS mechanical properties in non-industrial zone.

The loss of the adimensional stress and Young's modulus increases with the temperature and becomes increasingly important when approaching the thermoforming temperature. However, we note evolution of the adimensional elongation, which appea to indicate that the molecular chains are deformed, and the mechanical behavior of the material resembles that of caotechoutic material.

The comparison between the theoretical model (previously defined by Eqn. (3)) and experimental results, illustrated in Fig. 13exhibits strong agreement, which allows us to confirm the effectiveness of the model used in our study for the mechanical behavior of ABS material. 


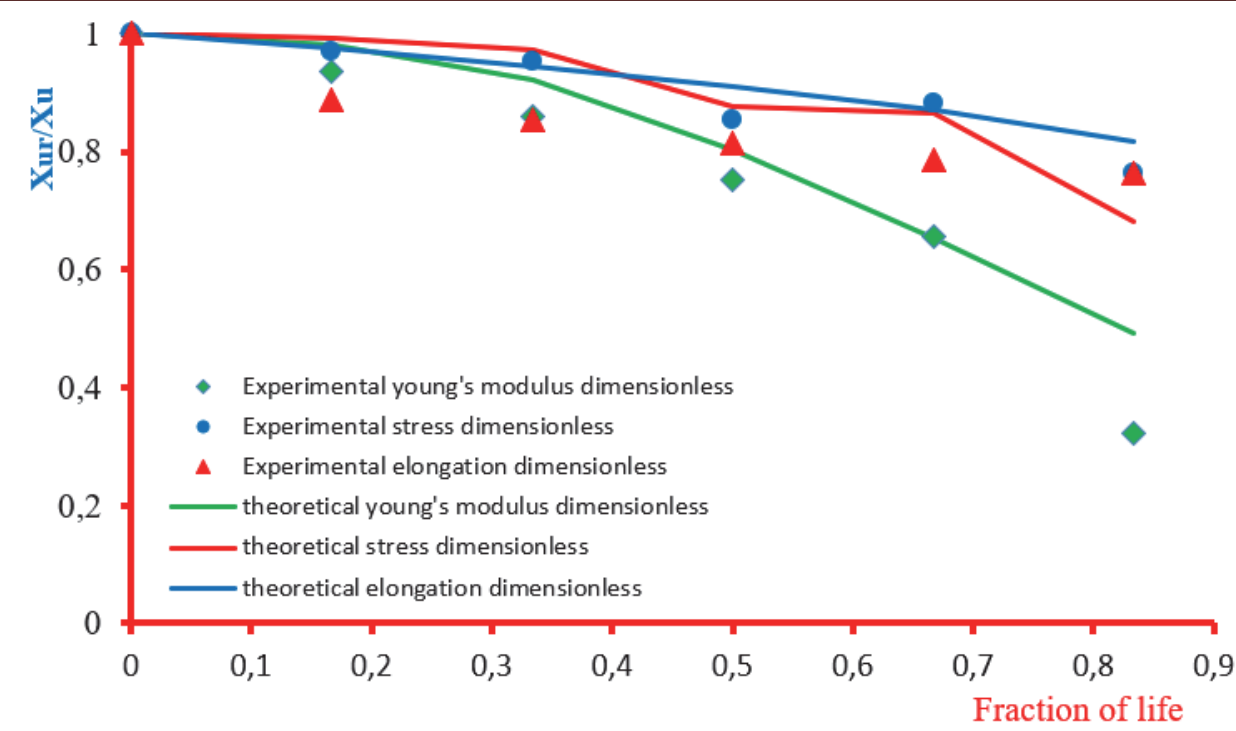

Figure 13: Theoretical and experimental adimensional losses of mechanical properties in non-industrial zone.

\section{Quantification of static flow}

As the study in this case is focused on the non-industrial zone, in which the temperature exceeds that of glass temperature, we no longer refer to the damage, but rather the flow.

The static flow based on changes in the ABS mechanical properties was developed to predict the evolution of the hightemperature polymer ( $\mathrm{T}>\mathrm{Tg})$. The static flow model is presented in Eqn. (8), [20].

$$
F=\frac{1-\frac{X^{\prime} u r}{X^{\prime} u}}{1-\frac{X^{\prime} a}{X^{\prime} g}}
$$

where:

X'ur :either residual ultimate stress ( $\left.\sigma^{\prime} u r\right)$, residual ultimate Young's modulus (E'ur) or residual ultimate elongation (L'ur); X'u :either ultimate stress ( $\sigma^{\prime} u$ ), ultimate Young's modulus (E'u) or ultimate elongation (L'u); and

X'a:either stress $\left(\sigma^{\prime} a\right)$, Young's modulus (E'a) or elongation (L'a) of the material directly before the end of its life.

Fig.14 illustrates the variation in the flow rate as a function of the fraction of life in the non-industrial zone.

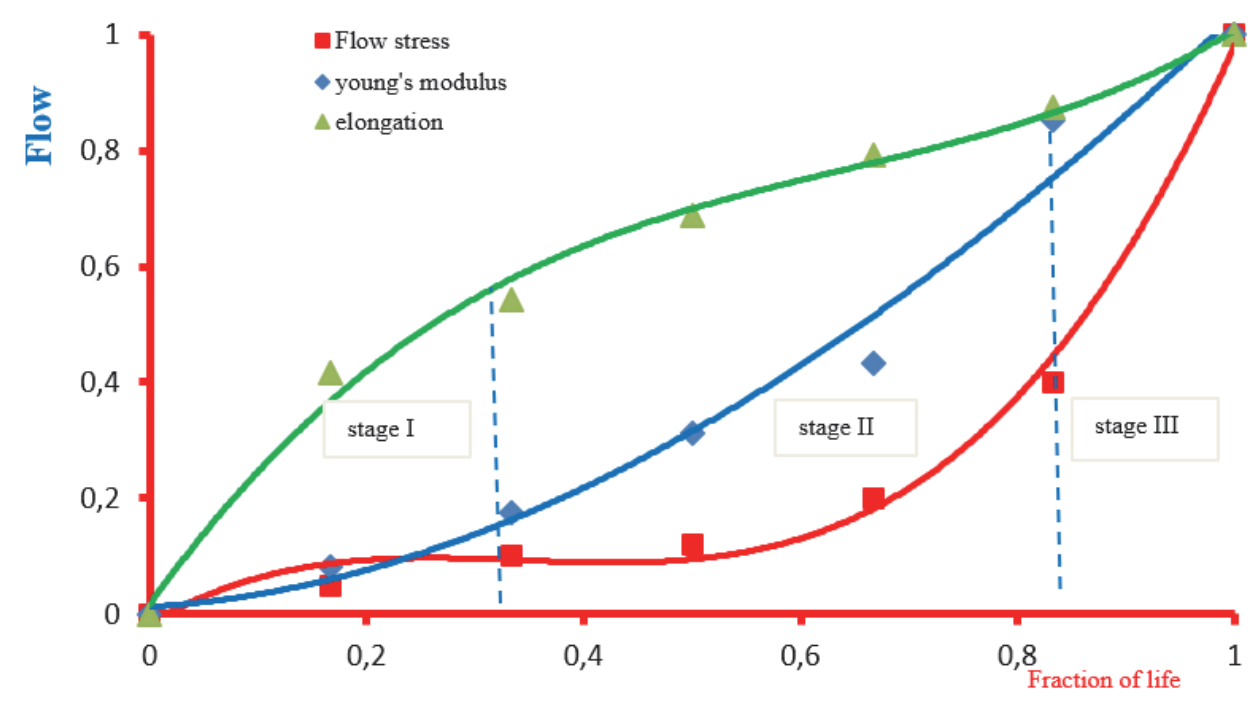

Figure 14: Evolution of flow as function of fraction of life $\beta$ ' in thermoforming zone 
The comparison of the ABS mechanical properties demonstrates that, for fractions with a short lifetime $(0 \%<\theta<30 \%)$, the flow calculated using the stress and Young's modulus is similar. With an increase of the fraction of life $\theta$, the stress flow curve is less significant compared to that calculated by the Young's modulus. Thereafter, it exceeds the stress curve and it is overlapped with the elongation curve at the end of the lifetime $\theta^{\prime}$. By observing the flow curves represented by the stress and Young's modulus, we can note the following characteristics:

* From the beginning of the flow to the end of stage I, in which the fraction of life is approximately $\theta^{\prime}=30 \%$, the flow increases linearly and slowly.

* In the progressive flow zone, namely stage $\Pi$, which is in the interval of $\theta^{\prime}=[30 \%, 85 \%]$, the flow increases concisely and progressively.

* At the end of the lifetime (stage $\amalg$ ), in which the fraction of life $\theta^{\prime}>85 \%$, the flow accelerates very clearly until the break.

\section{$>$ Relationship between static flow and static reliability}

The reliability varies inversely with the damage (in this case, the flow) [24]. Intuitively, a relationship must exist between these two parameters. This allows us to write:

$$
\operatorname{Rs}(\theta)+F(\theta)=1
$$

The resulting equation allows us to plot the variation in the reliability.

A decrease in the reliability means a loss of the material mechanical properties, and this loss evolves as the temperature becomes more important. The advantage of determining the reliability lies in the fact that it makes it possible to establish the critical damage in particular. Indeed, this information is crucial for preventive maintenance, in order to intervene in a timely manner and change the damaged part.

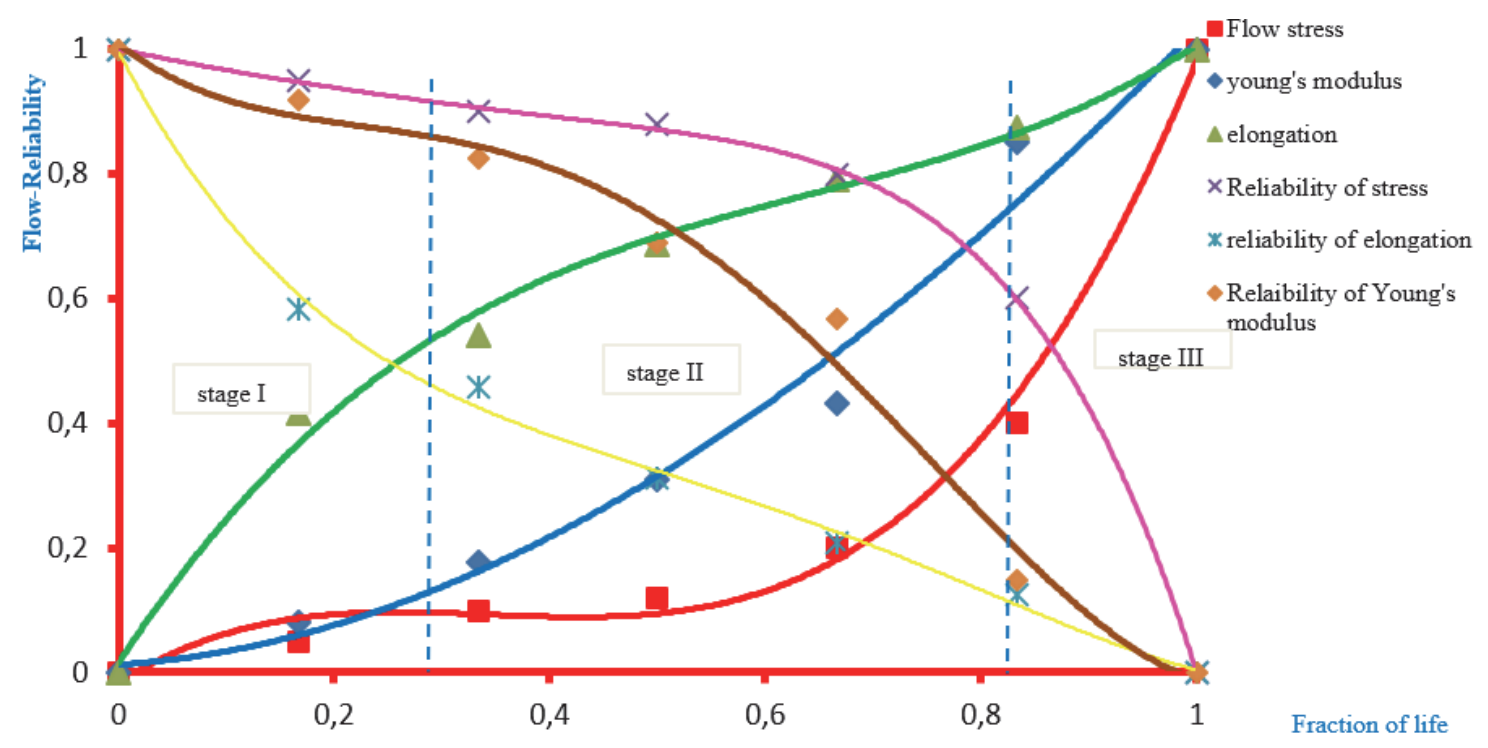

Figure 15: Evolution of flow with respect to reliability as function of fraction of life calculated using material parameters

Fig. 15 distinctly represents the evolution of the flow reliability as a function of fraction of life for each mechanical property parameter. Regardless of the parameter used, the flow gradually increases from 0 (vitreous temperature material) to its critical value of 1 . In fact, the increase in temperature induces a greater decrease in the ABS mechanical characteristics. This confirms the ability of this tensile mechanical characteristic to follow the degradation evolution reliably owing to the temperature increase. The paces calculated using the stress and elasticity modulus are similar they intersect at $50 \%$ reliability, with a relative difference for the fraction of life in the order of $10 \%$.The two models proposed in this study provide an overview of the flow condition and tend to overestimate the material loss. However, the flow reliability calculated by the elongation model does not describe the material state correctly, as there is no sophisticated means for exact determination of the elongation. We can conclude that the two models (stress and elasticity modulus) describe the loss of the studied material quite correctly: as the specimen temperature increases, its stability decreases and it becomes uncontrollable. 


\section{CONCLUSION AND OUTLOOK}

he objective of this work was to characterize the thermal and mechanical behavior of a thermoplastic flat sheet. The elevated temperature tensile test was considered as the most practical and accurate approach for the actual deformation state during loading, and this technique was used to describe the damage to the various thermomechanical characteristics: resistance, elasticity modulus, and elongation.

A comparison of the ABS mechanical characteristics enabled us to determine the performance of this material by exposing it to a tensile test coupled with temperature until the material was damaged. The evolution of the different parameters obtained allowed us to compare the temperature effects on the ABS mechanical behavior. Thereafter, we confirmed the ductile behavior of these, and we focused on comparing and processing the obtained curves. The increase in temperature demonstrated the critical impact on the material parameters and failure time. Indeed, the proposed approach consists of analyzing the evolution of the global geometry of the curves by considering the zones and characteristic points of these curves, and also takes into account the temperature effect, following the damage evolution by means of a static model. Two main zones were distinguished: the industrial zone, in which the configuration of the macromolecular chains was largely immobile and the temperature was below the glass temperature $\mathrm{Tg}=110^{\circ} \mathrm{C}$; and the thermoforming zone, in which the temperature was above the glass temperature $\mathrm{Tg}=110^{\circ} \mathrm{C}$. The macromolecular chains then tended to move more freely as the temperature increased, and thereafter the different stages of the process were determined.

Using the results of this work, we can construct a solid maintenance strategy and make the work with polymers more efficient and simpler. Moreover, the simplified approaches proposed allow for clients and industrial companies to evaluate the damage based on static tests alone, without performing dynamic tests. Furthermore, these can provide a tool that can aid in conducting rapid and relevant checks for the quality control of this material.

Finally, these results have demonstrated the feasibility of the applied damage approach. The proposed approach involves the material intrinsic parameters (elasticity modulus, elongation, and temperature), which enables a rigorous description of the material damage conditions.

These preliminary studies are essential steps for complete realization of our medium-term objectives for the implementation and development of modeling and simulation tools for thermoplastic forming processes.

\section{NOMENClATURE}

$\gamma e=\sigma e / \sigma a:$ Non-dimensional endurance limit

$\gamma \mathrm{u}=\sigma \mathrm{u} / \sigma \mathrm{a}$ : Parameter reflecting strength of material in virgin state

$\gamma=\sigma u r / \sigma a:$ Parameter characterizing effect of damage on material mechanical characteristics

ou: Endurance limit of the virgin material

our: Residual ultimate stress of material at different hole diameters

бa: Applied stress level

$\mathrm{m}$ : Material parameter

D: Damage ( $D=0$ for neat material, $D=1$ for completely damaged material)

$\beta$ : Life fraction for stress and Young's modulus in the industrial zone

$\beta^{\prime}$ : Life fraction for elongation in the industrial zone.

$\theta$ : Life fraction for stress and Young's modulus in the Thermoforming zone

$\theta^{\prime}$ : Life fraction for elongation in the Thermoforming zone

Tg: Glass transition temperature

Tf: Melting temperature

Ta: Ambient temperature

Ti: Instant temperature

\section{REFERENCES}

[1] Lligadas, G., Ronda, J. C., Galia, M. (2013). Renewable polymeric materials from vegetable oils: a perspective Caradiz Materials Today, 16(9). DOI: 10.1016/j.mattod.2013.08.016. 
[2] Christensen, R., (1980). Discussion: A Nonlinear Theory of Viscoelasticity for Application to Elastomers, Journal of Applied Mechanics, 47, pp. 682-683.

[3] Bui-Quoc, T., Dubuc, J., Bazergui, A., Biron, A., (1971). Cumulative fatigue damage under stress-controlled conditions, J. Basic Eng. Trans.

[4] Henry, D. L. (1955). A theory of fatigue damage accumulation in steel, Trans. Of the ASME, 77, pp. 913-918.

[5] Gatts, R. R., (1961). Application of cumulative damage concept to fatigue. ASME Journal of Basic Engineering. 83, pp. 529-540.

[6] Lemaitre, J., Chaboche, J.L., (1979), A Nonlinear Model of Creep-Fatigue Damage Cumulation and Interaction, National Office for Aerospace Studies and Research, pp. 1-30.

[7] Zgoul, M.H., Habali, S.M., (2008). An invistigation into pipes ashot water transporters in domestic and industrial applications, Jordan journal of mechanical and industrial engineering, 2, pp. $191-200$.

[8] Joseph, V. Rutkowski and Barbara, C. (1986). Acrylonitrile-Butadiene-Styrene Copolymers (ABS): Pyrolysis and Combustion Products and their Toxicity-A Review of the Literature; Levint us Department of Commerce, National Bureau of Standards, National Engineering Laboratory, Center for Fire Research, Gaithersburg, MD 20899

[9] Domínguez Almaraz, G. M. E., Correa Gómez, J.C., Verduzco Juárez, J.L., Avila Ambriz, (2015). Crack initiation and propagation on the polymeric material ABS (Acrylonitrile Butadiene Styrene), under ultrasonic fatigue testing; Avila Ambriz University of Michoacán (UMSNH), Santiago Tapia No. 403, Col. Centro, 58000, Morelia, Michoacán, Mexico.

[10] Bohatka, T. J., Moet, A.,(1995). Crack layer analysis of fatigue crack propagation in ABS polymer ;Department of Macromolecular Science, The Case School of Engineering, Case Western Reserve University, Cleveland, OH 44106, USA, 30(18), pp 4669-4675.

[11] Boldizar, A., Möller, K.,(2003). Degradation of ABS during repeated processing and accelerated ageing, Polymer Degradation and Stability, 81, pp. 359-366.

[12] Gibbs, J., (1961). The scientific papers of J. Willard Gibbs . Dover Publications, New York.

[13] Shen, M.C., Eisenberg, A.,(1966). Glass transitions in polymers, in progress in solid state chemistry, 3, Pergamon Press.

[14] ASTM D638-03 Standard test method for tensile properties of plastics.

[15] Rault, J., (2002). Les polymères solides, Amorphes, élastomères, semi-cristallins, Propriétés microscopiques et macroscopiques, Cépaduéséditions.

[16] Déformables, The ABS data sheet.

[17] Bathias, C., Bailon, J., (1980). The fatigue of materials and structures, pp. 328-330.

[18] Hicham, F., (2015). Behavior and damage of membranes thermoplastics in large deformations.

[19] Ladeveze, P., (1986). Damage mechanics for composite materials, 5ème journées nationales sur les composites, Paris.

[20] Abderrazak, En-Naji et al., (2019). Change Of Experimental Young's Modulus With Increasing Temperature For An Abs Material Subjected To Tensile Test; ARPN Journal of Engineering and Applied Sciences; 14(3), ISSN 1819-6608.

[21] Majid, F., et al., (2018). Continuum damage modeling throughtheoretical and experimental pressure limit formulas, Frattura ed Integrità Strutturale, 43, pp. 79-89; DOI: 10.3221/IGF-ESIS.43.05

[22] Ouardi, A., Majid, F. et alii. (2018). Residual life prediction of defected Polypropylene Random copolymer pipes (PPR), Frattura ed Integrità Strutturale, 43, pp. 97-105; DOI: 10.3221/IGF-ESIS.43.07.

[23] Majid, F., Elghorba, M. (2016). HDPE pipes failure analysis and damage modeling, DOI: $10.1016 /$ j.engfailanal.2016.10.002.

[24] Chapouille, P. et DePazzis, P., (1968). Fiabilité des systèmes, Editions Masson. 